Saint Louis University School of Law

Scholarship Commons

All Faculty Scholarship

2006

Conflicts of Interest in International Human Drug Research and the Insufficiency of International Protections

Robert Gatter

Follow this and additional works at: https://scholarship.law.slu.edu/faculty

Part of the Health Law and Policy Commons, and the International Law Commons 


\title{
Conflicts of Interest in International Human Drug Research and the Insufficiency of International Protections
}

\author{
Robert Gatter ${ }^{\dagger}$
}

\section{INTRODUCTION}

The world relies largely on private firms for the development of new medicine, and the system is efficient. Driven by the incentive to profit from sales of new pharmaceuticals, ${ }^{2}$ drug companies risk millions of dollars and years of work to shepherd basic scientific discoveries through laboratory and human testing in the hope of developing a marketable drug. For example, it is estimated that in 2002 alone, pharmaceutical companies invested $\$ 45$ billion the development of new medicine worldwide. ${ }^{3}$

While the profit incentive generates such enormous private investment in human drug development, it also encourages firms to pose inappropriate risks to the safety of human subjects when speeding a new drug to the market. The risks posed by financial conflicts of interest associated with human subjects research on new pharmaceutical products are notable examples, both in the U.S. and internationally. ${ }^{4,5}$ Many physicians who conduct such research, and research

Associate Dean for Academic Affairs and Professor of Law, Penn State University, Dickinson School of Law. I thank the editors and authors participating in this symposium for their invaluable comments on this essay.

See International Federation of Pharmaceutical Manufacturers and Associations (IFPMA), Position Statement, at http://www.ifpma.org/Issues/issues_research.aspx (claiming that the drug development investments of capital by private pharmaceutical firms are the most significant contribution to health research worldwide and that most of the world's existing medicines were discovered or developed through private investment).

In particular, the incentive is to make monopoly profits at least for the first 5 to 10 years a new drug is on the market based on the varying levels of patent protection afforded by national governments worldwide. See IFPMA, A Review of Existing Data Exclusivity Legislation in Selected Countries $\left(4^{\text {th }}\right.$ ed. 2005) (cataloging and summarizing patent protection law country by country), available at http://www.ifpma.org/News/Publications.aspx.

See IFPMA Position Statement, supra note 1.

For commentary addressing conflicts of interest in human subjects research in the U.S., see Robert Gatter, Walking the Talk of Trust in Human Subjects Research: The Challenge of Regulating Financial Conflicts of Interest, 52 EMORY L. J. 327 (2003); Jesse A. Goldner, Dealing with Conflicts of Interest in Biomedical Research: IRB Oversight as the Next Best Solution to the Abolitionist Approach, 28 I. L. MED. \& ETHICS 379 (2000); Richard S. Saver, Medical Research Oversight from the Corporate Governance Perspective: Comparing Institutional Review Boards and Corporate Beards, 46 WILliam \& MARY L. REV. 619, 621-633 (2004). 
institutions that house such research, have a financial interest in the outcome of the studies. Some own equity in the drug companies that own the products being tested and sponsor the studies to test those products on humans. ${ }^{6}$ More often, researchers and their institutions are the principal owners of "faculty start-up" companies, which hold the rights to the scientific discoveries that are being further developed through human testing, and which have entered, or anticipate entering, into licensing agreements with drug companies for further development. ${ }^{7}$ In both cases, researchers and their institutions have an incentive to see human testing go forward quickly and come to conclusions that tend to support the readiness of a new drug for market. The marketability of the drug increases the value of their equity holdings or the earnings that can be generated under a licensing agreement. Additionally, even when they do not have an ownership interest in the product being tested, researchers and research institutions have a financial incentive to serve the commercial interests of drug companies that sponsor human research because they rely on those companies to fund a substantial portion of their operating costs through the financial sponsorship of human drug research they conduct. ${ }^{8}$

Thus, for the sake of encouraging sponsorship of future human drug trials, researchers and their institutions are motivated to serve the interests of the drug companies sponsoring today's research. Moreover, financial conflicts of interest can be generated by paying researcher-physicians and third party physicians a fee for each human subject enrolled in a study, by paying fees to researchers for consulting with a drug company sponsoring a human drug trial conducted by those researchers, or by funding centers at the research institutions that house those trials.'

Although the U.S., the European Union (E.U.), and Japan are home to most of the world's private pharmaceutical developers, contain the largest consumer markets for human medicines, and operate the governmental agencies that approve or reject applications for new drugs to enter those markets, the conflicts of interest problems in human drug research span the globe. Drug companies increasingly test new drugs outside of the world's most industrialized countries, only to transfer back the test results to support applications in industrialized countries to market those new drugs. $^{10}$ Countries in Latin America, Eastern Europe, and Africa are just a few places that have become prime locations for privately sponsored human drug trials. ${ }^{11}$ Those countries generally offer a larger population of potential human subjects than available in industrialized countries, and they pose fewer regulatory barriers that tend to slow the progress of human drug trials. ${ }^{12}$

For commentary addressing conflicts of interest in human subjects research internationally, see Robert Gatter, Financial Conflicts of Interest in Human Subjects Research: Domestic and International Issues, in ANNALS OF BIOETHICS (Sandra H. Johnson, ed.) (forthcoming) (manuscript available upon request).

Id. at 334-344.

Id.

Id. at 346-348.

Id. at $344-348$

See Janet Rehnquist, Office of the Inspector General, U.S. Department of Health and Human Services, The Globalization of Clinical Trials: A Growing Challenge in Protecting Human Subjects, available at http://oig.hhs.gov/oei/reports/oei-01-00-00190.pdf (Sept. 2001). See also Gatter, supra note 5, (manuscript at 7-8).

$11 \quad I d$. at 8.

12 Id. at 7-8. 
As drug companies outsource their human research to developing countries, they also export the financial conflicts of interest problem. ${ }^{13}$ For example, a December 2000 investigative report by the Washington Post revealed that drug companies often double or triple the annual incomes of health professionals in developing countries by paying salaries and human subject recruitment fees that are moderate under U.S. standards. ${ }^{14}$ Similarly, the report established that governments in developing countries are eager to host human drug trials sponsored by pharmaceutical companies as a way of infusing their health care delivery systems with money. ${ }^{15}$

Financial ties among researchers, research institutions and the host country governments, on the one hand, and private firms sponsoring human drug research, on the other hand, create an incentive for researchers, institutions and governments to serve the commercial interests of the sponsoring drug companies. This conflicts with their interest to assure the safety of human subjects in those trials. For example, a researcher with a financial interest in conducting a quick and successful human trial has an incentive to enroll a subject even when the subject might not meet all of the clinical criteria for participation, or to underestimate the importance of a subject's adverse reaction to participation. Likewise, institutions with a financial interest in the studies they house have an incentive to permit those studies to go forward despite evidence of potentially inappropriate risks to human subjects. Similarly, governments eager to encourage drug companies to conduct future human trials in their countries have an incentive to encourage national and local committees designed to protect the safety of human subjects to be lenient in their oversight of the research.

Indeed, the Post investigation found that human subjects in trials conducted in foreign countries were exposed to inappropriate risks when researchers deviated from research protocols, adverse findings were not reported, potential human subjects were not adequately informed of the risks of participation, and enrolled human subjects whose conditions worsened were not moved from experimental to standard treatments. ${ }^{16}$ Additionally, a 2001 report from the Office of the Inspector General of the U.S. Department of Health and Human Services found that ethics committees responsible for assuring human subject safety in foreign clinical trials often were too disorganized to sufficiently serve their purpose. ${ }^{17}$ This is particularly troubling because international guidelines on human subject research and the laws in each of the world's major drug-producing markets rely heavily on researchers and ethics committees to protect the safety of human subjects. ${ }^{18}$

Id. at 8 ("As manufacturers in the major drug-producing economies outsource clinical trials to foreign countries, they also export financial COls and the risks those COls pose to human subject safety").

See Joe Stephens, The Body Hunters: Exporting Human Experiments (pt. 1), WaSH. POST, Dec. 17, 2000, at A J; Mary Pat Flaherty et al., The Body Hunters: Overwhelming the Watchdogs (pt. 2), WASH. POST, Dec. 18, 2000, at Al.

15 Flaherty et al., supra note 14 , at A1.

16 See Stephens, supra note 14; Flaherty, supra note 14

17 See Rehnquist, supra note 10 , at $12-13,15$.

18 For international guidelines, see Council for International Organizations of Medical Sciences (2002) [hereinafter CIOMS], INTERNATIONAL ETHICAL GUIDELINES FOR BIOMEDICAL RESEARCH INVOLVING HUMAN SUBJECTS http:/www.cioms.ch/frame_guidelines_nov_2002.htm; WHO, GUIDELINES FOR GOOD CLINICAL PRACTICE (GCP) FOR Trials on PharmaceutiCal Products (I995); International Conference on Harmonisation, Guidelines FOR GOOD CliniCal PRACTICE (1996), http:/www.ich.org/LOB/media482.pdf. For domestic laws, see, e.g., 45 C.F.R. pt. 46 (U.S. 
Thus, the problem of financial conflicts of interest in human subjects research is international in scope, and poses a substantial challenge for international regulation. This essay identifies international standards available to protect human subjects from the risks associated with financial conflicts of interest in human drug trials and assesses their adequacy. Given the rise in foreign drug trials-i.e. human drug research sponsored by a pharmaceutical company based in a highly industrialized country, but conducted in a developing country - such trials are the exclusive focus of the essay.

\section{INTERNATIONAL STANDARDS CONCERNING CONFLICTS OF INTEREST IN HUMAN SUBJECTS RESEARCH}

There are four major sources for international standards for human drug research. First is the Helsinki Declaration, which was crafted by the World Medical Association in 1964, and has been amended five times since, including most recently in 2000. ${ }^{19}$ The Helsinki Declaration establishes the basic ethical principles and procedures for conducting human medical experiments and is regarded as the fundamental document on point. ${ }^{20}$ The other three major sources were derived from the Helsinki Declaration. ${ }^{21}$ These sources are: the Guidelines for Good Clinical Practice for Trials on Pharmaceutical Products, adopted by the World Health Organization (the "WHO GCPs") in $1995,{ }^{22}$ the Guidelines for Good Clinical Practice, adopted by the International Conference on Harmonisation of Technical Requirements for Registration of Pharmaceuticals for Human Use (the "ICH GCPs") in $1996,{ }^{23}$ and the International Ethical Guidelines for Biomedical Research Involving Human Subjects, adopted by the Council for International Organizations of Medical Sciences (the "CIOMS Guidelines") in 1993 and amended in 2002.

Because each of these three sources evolved out of the Helsinki Declaration, it is not surprising that each establishes a very similar method for assuring the safety of human subjects in drug trials. Generally speaking, they each rely on the oversight of an ethics committee, together with the informed consent of human subjects and the cooperation of researchers to continuously assure the safety of human subjects.

regulations); Directive on Clinical Trials (2001/20/EC) of the European Parliament and the Council of the European Union (2001), available at http://eudract.emea.eu.int/docs/Dir2001-20_en.pdf. According to an international survey of laws related to human subjects research recently published by the U.S. Office for Human Research Protections in the Department of Health and Human Services, Japan has adopted the ICH's Good Clinical Practice Guidelines as law. See OHRP, International Compilation of Human Subjects Research Protections (2d ed. Oct. 1, 2005), available at http://www.hhs,gov/ohrp/international/HSPCompilation.pdf.

19 See World Medical Association, Declaration of Helsinki, reprinted in, CIOMS, INTERNATIONAL ETHICAL GUIDELINES FOR BIOMEDICAL RESEARCH INVOLVING HUMAN SUBJECTS 89 (2002) (App. 2), http://www.wma.net/e/policy/b3.htm.

20 The Helsinki Declaration was itself an effort to describe in more detail the general statements in the Nuremberg Code of 1947 and the Universal Declaration of Human Rights of 1948 that humans shall not be subject to medical experimentation without their consent. See CIOMS, INTERNATIONAL ETHICAL GUIDELINES FOR BIOMEDICAL RESEARCH INVOLVING HUMAN SUBJECTS 15 (2002).

See CIOMS, supra note 18, (Discussing three notable guideline publications by international organizations since the publication of CIOMS 1993 Guidelines).

${ }_{22}$ WHO, Guidelines for Good Clinical Practice for Trials on Pharmaceutical Products, in The Use of ESSEnTIAL DrugS 97-137 (Technical Report Series No. 850)(1995)(Annex 3) (WHO's guidelines were originally published as an appendix to this technical report).

${ }_{23}$ See International Conference on Harmonisation, GUIDELINES FOR GOOD CLINICAL PRACTICE (1996), http:/www.ich.org/LOB/media/MEDIA482.pdf.

24 See CIOMS, supra note 18. 
Despite the general similarity of these leading sets of guidelines, they address conflicts of interest differently. There are several issues to consider when comparing the guidelines on matters related to conflicts of interest. The first issue is the scope of a guideline, namely whether it addresses any conflicts of interest at all, addresses only those of committee members who review the ethical propriety of proposed research, or addresses the conflicts of interest of committee members and researchers alike. The second issue is whether the guideline offers any substantive limits or prohibitions, or whether it recommends only the procedural protections of disclosure. If the latter is true, then the additional question exists of whether disclosure is made only to review committees or to prospective human subjects as well. The final issue for comparison is whether a guideline addresses the likelihood that countries will regulate conflicts of interest differently and addresses the risk of "forum shopping" among human drug trial sponsors and research. Specifically, the issue is whether a guideline accounts for the risk that human drug trial sponsors and researchers will take advantage of more lenient regulations in a foreign country and use financial incentives in the hopes of completing a trial more quickly.

Comparing the CIOMS, WHO and ICH guidelines on these issues reveals a range of conflict-of-interest protections, from the moderate protection afforded by the CIOMS Guidelines to the virtually non-existent protection of the ICH GCPs. Additionally, it suggests that none of the guidelines are sufficient to adequately protect human subjects from the risks associated with conflicts of interest in human drug research.

Before comparing the three leading, modern guidelines, it is worth examining the conflicts of interest protections proposed by the Helsinki Declaration, from which each of the modern guidelines is derived. ${ }^{25}$ Rather than prohibiting conflicts of interest, the Helsinki Declaration recommends that human research not be conducted unless it has first been reviewed and approved by an "ethical review committee" that is "independent of the investigator, the sponsor or any other kind of undue influence." 26 Moreover, it suggests that the committee's review should encompass examining whether researchers have any conflicts of interest. ${ }^{27}$ Under the Declaration, researchers "should also submit to the committee, for review, information regarding funding, sponsors, institutional affiliations, and other potential conflicts of interest and incentives for subjects." ${ }^{28}$ It also envisions that prospective human subjects will be told of "any possible conflicts of interest" as part of the informed consent process. ${ }^{29}$ Finally, the Declaration instructs researchers who publish the study results to publish negative as well as positive findings and to declare "funding, institutional affiliations and any possible conflicts of interest" in the publication. ${ }^{30}$

25 This examination is based upon the current version of the Helsinki Declaration. The conflicts of interest provisions of the declaration appear to have been added in 2000 (Edinburgh, Scotland) because they do not appear in the 1996 version.

26 Declaration of Helsinki, supra note 19 , at 91 , para. 13.

2? Id. ("The researcher should also submit to the committee, for review, information regarding funding, sponsors, institutional affiliations, other potential conflicts of interest and incentives for subjects.")

See id. at 92 para. 22.

so $\quad$ ld. at $93-94$ para. 27. 


\section{A. CIOMS}

Of the three modern guidelines, those adopted by CIOMS offer the greatest protection against the risks posed by conflicts of interest. First, the CIOMS Guidelines directly acknowledge the problem of conflicts of interest and recommend that researchers avoid entering into arrangements that "interfere unduly" with their ability to access or analyze data or to publish research results. ${ }^{31}$ While they do not prohibit or set substantive limits on conflicts of interest, the CIOMS Guidelines attempt to account for them through various procedural safeguards. First, they recommend that researchers "disclose potential or apparent conflicts of interest on their part to the ethical review committee or to other institutional committees designed to evaluate and manage such conflicts." 32 Second, to assure the independence of the ethical review committee, the guidelines propose the same disclosure requirement for members of the ethical review committee, and also recommend that committee members not be permitted to participate in the review of a protocol in which they have an interest sufficient to "subvert the member's objective judgment." 33 Third, the CIOMS Guidelines clearly recognize the risk that researchers and sponsors forum shop, so as to conduct research in countries with less stringent regulatory protections than those in their home countries. ${ }^{34}$ To address this risk, they recommend that research protocols undergo ethical review "in the country of the sponsoring organization, and [that] the ethical standards applied ... be no less stringent than they would be for research carried out in that country." 35

According to the issues outlined above, the CIOMS Guidelines offer a moderate degree of protection from the risks associated with conflicts of interest. They address the risk directly and attempt to account for it among both researchers and members of ethics committees that review proposed human drug trials. The proposed rules are procedural rather than substantive, requiring that researchers and review committee members disclose potential conflicts of interest to the review committee, which presumably determines whether any undue influence exists. ${ }^{36}$ Moreover, the Guidelines recognize and address the risk that sponsors and researchers take advantage of lax conflicts-of-interest regulation in developing countries by proposing the application of standards from the sponsor's home country even when the trial will be conducted overseas.

Yet, the protections afforded under the CIOMS Guidelines also have significant limitations. Most notably, they do not define what constitutes a conflict of interest, nor do they set any substantive standard for when a conflict of interest is unacceptable. Thus, they allow researchers, sponsors and ethics committees to determine for themselves where substantive lines should be drawn. This is troublesome because drug companies who sponsor human trials may have a great deal of influence over the members of ethics committees, particularly those in developing countries. While the Guidelines recognize that ethics committee members can have financial interests in a protocol under review significant enough

\footnotetext{
See CIOMS, supra note 24 , at 29-30 (commentary to guideline 2).

See id. at 27 (commentary to guideline 2).

Id.
}

In the guideline's introductory statement of general ethical principles, it notes that researchers and sponsors should not "take advantage of the relative inability of low-resource countries or vulnerable populations to protect their own interests, by conducting research inexpensively and avoiding complex regulatory systems of industrialized countries . . ." Id. at 18 .

35 CIOMS, supra note 24 , at 31 (guideline 3 ).

36 See id. (commentary to guideline 6). 
to prohibit the member from participating in the protocol's review, they also acknowledge that ethics committees may be "institutional." "37 This appears to authorize the kind of ethics committees utilized in the U.S., which are composed almost entirely of individuals employed by the research institution that will house the studies being reviewed by the ethics committee (known as an "institutional review board" or "IRB"). Such a committee structure automatically introduces a financial bias into the review of proposed human drug trials because the research institution, as the entity housing the trial, will receive funds from a sponsoring drug company if the institution's review committee approves the trial. Accordingly, a committee consisting of institutional insiders is likely to be biased in favor of approving such proposed research.

Moreover, sponsors of human drug trials in developing countries may have even more influence over the ethics committees that review their proposed research because those sponsors may indirectly fund the committees. ${ }^{38}$ The CIOMS Guidelines anticipate that countries in which human research will be conducted may not have any ethics committee review system in place. In such a circumstance, the Guidelines impose on research sponsors the obligation to help fund the creation of such a system. ${ }^{39}$ Despite the expectation that such funding will be provided to the public authorities that create the review committee and not directly to the committee itself, ${ }^{40}$ it is not difficult to imagine that even such indirect financing of the ethics committee by a research sponsor risks biasing the review process in favor of the sponsor. Thus, by permitting financial bias in the structure of ethics committees while also proposing a system recusing committee members with potential conflicts of interest, the CIOMS Guidelines send mixed signals. Any assessment of the protection those Guidelines afford with respect to conflicts of interest must be downgraded accordingly.

Finally, the protection provided by the CIOMS Guidelines is further limited because human subjects are not told of the researchers' or review committee members' potential conflicts of interest. Although no person may participate in a human drug trial without giving informed consent of the risks and benefits of participation, the Guidelines do not impose a duty to disclose conflicts of interest. ${ }^{41}$ At most, prospective human subjects must be informed of "the sponsors of the research, the institutional affiliation of the investigators, and the nature and sources of funding for the research."42 At best, such information will only give clues about researchers' potential conflict of interest, and will provide almost no information about the financial interests of the institution or the review committee members. Thus, the Guidelines fail to take advantage of the protection afforded through a more rigorous informed consent process.

\section{B. WHO GCPS}

The WHO GCPs offer even less protection against the risks associated with conflicts of interest than do the CIOMS Guidelines. Indeed, the phrase "conflict of interest" does not appear anywhere in the GCPs. The only protection comes from a

See id. at 24 (commentary to guideline 2).

See id. at Appendix 1.

See id. at 80-81 (guidelines 20 and its commentary).

$I d$ at 81 (commentary to guideline 20).

See id. at 39 (guideline 5).

Id. 
proposal requiring disclosure of financial relationships between researchers and sponsors in human drug protocols to the "independent" ethics committees that will review those protocols and to governmental agencies with jurisdiction over the approval of new drugs. ${ }^{43}$ While this suggests that ethics committees will account for researchers' conflicts of interest, a question exists about the relative independence of the ethics committee itself under the WHO GCPs, just as there is under CIOMS Guidelines. An "independent ethics committee" is defined as a committee "free from bias and from any influence of those who are conducting the trial." 44 It is unlikely, however, that the language "free from . . . any influence" is as absolute in practice as it sounds because WHO GCPs also define "ethics committee" to include an "institutional" committee. ${ }^{45}$ As explained above, an institutional committee likely encompasses U.S.-style IRBs, which are comprised almost exclusively of individuals employed by the institution that would house the research under consideration and receive financial support from the study's sponsor for doing so. Accordingly, the protection afforded to human subjects from the risks associated with researchers' conflicts of interest through a process of disclosure and review by an ethics committee must be discounted by the fact that the ethics committee could be operating under its own conflict of interest.

Moreover, the WHO GCPs provide no other protections to supplement the process of disclosure and review by an ethics committee. For example, like the CIOMS Guidelines, the WHO GCPs do not attempt to regulate conflicts of interest substantively by prohibiting certain kinds of financial interests. ${ }^{46}$ Also like the CIOMS Guidelines, they do not impose, as part of the informed consent process, an obligation to disclose to prospective human subjects conflicts of interest or even information about financial relationships among researchers, sponsors, institutions and review committee members. ${ }^{47}$

Yet, the paucity of protections in the WHO GCPs goes beyond that of the CIOMS Guidelines. For example, the WHO GCPs do not attempt to enforce a standard of independence among those responsible for reviewing proposed human subject trials. So, while there is a requirement for researchers to disclose their financial interests to an ethics committee for review, and there is an expectation that such a committee will act in an "independent" manner, there is no mechanism proposed to assure the independence of the ethics committee. ${ }^{48}$ Furthermore, the WHO GCPs completely fail to address the risk of forum shopping among developing countries by sponsors and researchers in search of lenient regulations. Thus, according to the issues outlined above, the WHO GCPs offer, at best, only modest protection for human subjects from conflicts of interest, and substantially less than those provided under CIOMS Guidelines.

\section{ICH GCPS}

Despite their relatively weak showing, the WHO GCPs do not fare the worst in the comparison of conflicts of interest standards among today's leading international statements related to human subjects research. That distinction goes to the ICH

See WHO, supra note 22, at 115 (sec. 2.11 ).

Id. at 103 .

Id.

See generally WHO, supra note 22.

See Id. at 110-112 (sec. 3.3), 136-137 (Appendix 2) for informed consent disclosures.

See generally WHO, supra note 22 . 
GCPs, which like the WHO GCPs, do not attempt to regulate conflicts of interest in any substantive way through prohibitions or limitations on financial relationships among researchers, sponsors, institutions and ethics committees. ${ }^{49}$ In keeping with WHO GCPs, the ICH GCPs also do not require disclosure of conflicts of interest information to prospective human subjects as part of the informed consent process. Moreover, they do not address the risk that loose or non-existent regulation concerning conflicts of interest in many developing countries creates improper incentives for sponsors to conduct human drug trials in those countries to cash-in on the absence of such rules. The ICH GCPs, however, contain an additional gap in the management of conflicts of interest that does not exist in either the CIOMS Guidelines or the WHO GCPs. While the ICH GCPs require approval of a human drug trial by an "independent ethics committee" before the trial is commenced, the guidelines do not require providing the ethics committee with any information concerning the potential or actual conflicts of interests of researchers. ${ }^{50}$ They expect that "financial aspects of the trial" will be "documented in an agreement between the sponsor and the investigator/institution," 51 but there is no affirmative obligation that the agreement be disclosed to the ethics committee. ${ }^{52}$ Simply put, an ethics committee cannot protect human subjects from risks associated with conflicts of interest about which it has no information.

If the Helsinki Declaration and the three leading, international sets of guidelines are ranked from the most to least protective (Table 1), the Helsinki Declaration and the CIOMS Guidelines finish at the top of the list, followed by the WHO GCPs, and then by the ICH GCPs. At the same time, it is clear that each of the guidelines falls short of providing ideal protection for human subjects from conflicts of interest because each fails to regulate conflicts of interest substantively or to recommend that individual states do so.

t9 See Finnuala Kelleher, The Pharmaceutical Industry's Responsibility for Protecting Human Subjects of Clinical Trials in Developing Nations, 38 COLuM. J.L. \& SoC. ProbS. 67, 78. See generally Cinead R. Kubiak, Conflicting Interests \& Conflicting Laws: Re-Aligning The Purpose And Practice Of Research Ethics Committees, 30 BROOKLYN J. INT'L L. 759, 784-786 (2005).

so See Kubiak supra note 49 , at 808 (quoting "Unfortunately, since the [ICH] GCP mirrors U.S. human-subject research regulations, it also carries the same flaws... The ICH GCP requires documentation of financial arrangements among investigators, institutions and sponsors. Yet, these documents are only required to be maintained by the sponsor and research institution. As with the 1989 Declaration and U.S. regulations, the ICH GCP does not require that RECs are provided COI disclosure statements").

51 See ICH, supra note 23, at sections 4.9.6 and 5.9.

52 The guidelines provide that the protocol should address "financing and insurance" if not addressed in a separate agreement, see $\mathrm{ICH}$, supra note 23 , at sec. 6.14 , but it is unclear whether this very vague language is meant to encompass conflicts of interest. Additionally, given that there is no statement that a separate agreement concerning finances should be made part of a protocol submitted to an ethics committee, the request for information on "financing and insurance" may in effect be optional because the information could always be put in a separate agreement that does not appear to be subject to review. 


\begin{tabular}{|l|l|l|l|l|l|}
\hline TABLE 1 & $\begin{array}{l}\text { Substantive } \\
\text { Regulation }\end{array}$ & $\begin{array}{l}\text { Researcher } \\
\text { Disclosure }\end{array}$ & $\begin{array}{l}\text { Committee } \\
\text { Member } \\
\text { Disclosure/ } \\
\text { Recusal }\end{array}$ & $\begin{array}{l}\text { Disclosure to } \\
\text { Subject }\end{array}$ & $\begin{array}{l}\text { Addresses } \\
\text { Forum } \\
\text { Shopping }\end{array}$ \\
\hline Helsinki & & $\bar{X}$ & $\mathrm{X}$ & $\mathrm{X}$ & \\
\hline CIOMS & & $\mathrm{X}$ & $\mathrm{X}$ & & $\mathrm{X}$ \\
\hline WHO GCPs & & $\mathrm{X}$ & & & \\
\hline ICH GCPs & & & & & \\
\hline
\end{tabular}

One possible reason for the disparity among these international sources for human research standards is that the public has only recently turned its attention to financial conflicts of interest in human subjects research due to several high profile cases. $^{53}$ For example, Jesse Gelsinger died while participating in a gene therapy study conducted at the University of Pennsylvania in $1999 .^{54}$ The claim that the death may have been caused by misconduct allegedly motivated by the financial interests of both the lead researcher and the University were widely publicized. ${ }^{55}$ Thus, those drafting the WHO and ICH GCPs in the mid-1990s may not have considered including conflict of interest standards because conflicts of interest in human research had yet to become a burning public issue. In comparison, the most recent revisions of both the Helsinki Declaration and the CIOMS Guidelines were published in 2000 and 2002 , respectively, ${ }^{56}$ and so their drafters had the opportunity to reconsider international standards following public recognition of the threat that conflicts of interest might pose to human subjects. Indeed, if all four international statements were re-organized according to their year of publication (Table 2 below), there appears to be a relationship between the degree of protection afforded in a given source and whether or not that source was published before 1999 .

\begin{tabular}{|l|c|l|c|l|l|l|}
\hline TABLE 2 & Date & $\begin{array}{l}\text { Substantive } \\
\text { Regulation }\end{array}$ & $\begin{array}{l}\text { Researcher } \\
\text { Disclosure }\end{array}$ & $\begin{array}{l}\text { Committee } \\
\text { Member } \\
\text { Disclosure/ } \\
\text { Recusal }\end{array}$ & $\begin{array}{l}\text { Disclosure } \\
\text { to Subject }\end{array}$ & $\begin{array}{l}\text { Addresses } \\
\text { Forum } \\
\text { Shopping }\end{array}$ \\
\hline WHO GCPs & 1995 & & $\mathrm{X}$ & & & \\
\hline ICH GCPs & 1996 & & & & & \\
\hline Helsinki & 2000 & & $\mathrm{X}$ & $\mathrm{X}$ & $\mathrm{X}$ & \\
\hline CIOMS & 2002 & & $\mathrm{X}$ & $\mathrm{X}$ & & $\mathrm{X}$ \\
\hline
\end{tabular}

Despite the way these international sources for human research standards align themselves in Table 2, however, the publication date cannot fully explain the

53 See Saver, supra note 4, at 621-626 (referring to several highly publicized incidents of conflicts of interest related to human subjects research from 1999 through 2001 ).

54 See Gatter, supra note 5, at 329-330.

s5 See id.

s6 See The World Medical Association's organization definition of the Declaration of Helsinki on their website, http://www.wma.net/e/ethicsunit/helsinki.htm. 
different levels of protection from conflicts of interest offered by each. For example, the WHO GCPs pre-date those of ICH by one year. Thus, the WHO standard requiring researchers to disclose their financial relationships with sponsors to the reviewing ethics committee was available to $\mathrm{ICH}$ at the time it drafted its GCPs. This suggests that silence in the ICH GCPs on conflicts of interest might be deliberate rather than accidental. Even more striking is the fact that an earlier version of the CIOMS Guidelines, published in 1993, included many of the same standards relating to conflicts of interest that appear in the current version published in 2002 and described above. ${ }^{57}$ Thus, it appears that both WHO and ICH should have been aware of the problem posed by conflicts of interest at the time each was drafting its most recent GCPs, again suggesting that the lack of standards relating to conflicts of interest in both the WHO and ICH GCPs resulted from a deliberate choice. Finally, the governments overseeing the three largest drug-producing and drug-consuming markets (the U.S., E.U and Japan) each adopted the ICH GCPs as law or as guidelines in their respective countries within about one year of the GCPs' publication by $\mathrm{ICH}$ in $1996 .^{58}$ Thus, those governments endorsed the guidelines that offer the least protection to human subjects against the risks associated with conflicts of interest, despite the existence of WHO GCPs and the 1993 version of CIOMS Guidelines. Both of which suggested more strict regulation of conflicts of interest than the ICH GCPs. Likewise, the Council of Ministers of the EU - the body with governing authority to direct EU members to pass regulations on matters affecting the Union - issued a directive in 2001 requiring EU member countries to adopt a set of good clinical practices approved by the Council. ${ }^{59}$ Those practices implement standards that, at most, offer only the protection afforded in the WHO GCPs. They require approval of human drug trials by an independent ethics committee, which is authorized to give its opinion about "the amounts and, where appropriate, the arrangements for rewarding or compensating investigators . . . and the relevant aspects of any agreement between the sponsor and the site." ${ }^{60}$ While the EU directive does not specifically require researchers to report their financial arrangements with sponsors and institutions, the power to discover such information is implicit in the power to render an opinion about the propriety of those arrangements. ${ }^{61}$ No other standards are required that relate to conflicts of interest in the directive.

In short, given that non-governmental and governmental agencies have repeatedly chosen to adopt human research standards that provide only minimal, if any protection for human subjects against the risks associated with conflicts of interest, and given that they have done so despite the existence of more protective standards, it appears that the international community may be deliberately choosing to provide less protection against conflicts of interest. This possibility is even more

57 See CIOMS, International Ethical Guidelines for Biomedical Research Involving Human Subjects, Guidelines 8, 14, 15 and their commentary (1993), http://www.fhi.org/training/sp/RETC/cioms.htm.

58 See ICH Implementation Grid, http://www.ich.org/cache/compo/276-254-1.html. In the U.S., the Food and Drug Administration adopted the ICH GCPs as an industry guidance in 1997. See Good Clinical Practice: Consolidated Guideline, 62 Fed. Reg. 25692 (May 9, 1997). And in 2004, proposed making those conditions into mandatory standards. See Foreign Clinical Studies Not Conducted Under an Investigational New Drug Application, 69 Fed. Reg. 32467 (June 10, 2004).

59 See Council of Ministers of the EU, Directive on Clinical Trials, Directive 2001/20/EC, L

121 OfFICIAL JOURNAL OF THE EUROPEAN COMMUNITIES 34 (May 1, 2001), available at http://europa.eu.int/eur-lex/pri/en/oj/dat/2001/1_121/1_12120010501en00340044.pdf.

60 See id. at Art. 6, 9 3(j).

61 Id. 
likely when one considers the political and economic context in which international human research standards were crafted.

Facilitating the private development of new human medicines and their efficient distribution throughout the world has been the primary interest driving international standards for human drug research. This is particularly true of GCPs adopted by WHO and ICH in the mid-1990s. Indeed, ICH is a cooperative venture by representatives of the pharmaceutical industries and drug regulators in the U.S., E.U and Japan. ${ }^{62}$ ICH was designed to create uniform regulations worldwide for the marketing of new human medicines so that a drug company would only need to satisfy one set of requirements in order to market its products in each of the world's major drug markets. ${ }^{63}$ Likewise, the WHO GCPs were devised "as a contribution to harmonization of national standards and to facilitating the movement of pharmaceutical products internationally." 64

Given their aim to promote uniformity and international commerce in pharmaceuticals, WHO and ICH, at the time they each crafted their GCPs, had an incentive to adopt only minimal regulatory barriers and to require the least change from customary regulatory practices. Thus, neither project sought to dramatically strengthen human subject protections. Instead, each was "based on provisions already promulgated in a number of countries, including . . European Community countries, Japan, ... and the United States." Therefore, "care [was] taken, in developing the present Guidelines . . . to assure their compatibility with existing national and other provisions." ${ }^{66}$ In other words, given a choice among conflicting international standards, ICH and WHO consciously favored standards that enabled drug companies to bring their products to the international market quickly and required the least regulatory change. Accordingly, it is not surprising that $\mathrm{ICH}$ and WHO would ignore more strict standards related to conflicts of interest that were proposed by CIOMS in I993. In the end, international standards for the protection of human subjects in drug trials appear heavily influenced by the commercial interests of the international pharmaceutical industry. This has resulted, at least initially, in relatively lax international standards for conflicts of interest in human drug trials.

\section{CONCLUSION: THE FUTURE OF INTERNATIONAL CONFLICTS OF INTEREST STANDARDS}

Various international standard-setting projects related to human subjects research are ongoing. Thus, we are likely to see more revisions of guidelines from WHO, ICH, CIOMS and perhaps from other organizations as well. There are likely to be opportunities to strengthen international standards related to conflicts of interest in human drug trials.

Yet, given the commercial interests at stake and the fact that they appear to have driven recent international, standard-setting efforts, one cannot assume that conflicts of interest standards will be strengthened. Rather, the safer assumption is that organizations such as ICH must be pressured into adopting stronger conflicts of interest protections. The question for the future is how to best apply such pressure.

62 ICH Implementation Grid, supra note 58.

63 See ICH Home Page, http://www.ich.org/cache/compo/276-254-1.html.

64 WHO, supra note 22 , at 100.

65 Id.

$66 \quad I d$. 
There are several strategies to employ that may generate the political will for organizations to adopt stronger conflicts of interest protections, but each has its own limitations. For example, CIOMS could take a public stand for the need to bring existing international standards in line with those standards that CIOMS has proposed since at least 1993. Unfortunately, CIOMS has not taken advantage of the earlier opportunities to do so. ${ }^{67}$ For example, the introductory materials to the 2002 CIOMS Guidelines acknowledges other international standards related to human subjects research, but never makes a comparison between its Guidelines and those promulgated by other organizations. ${ }^{68}$ This leaves the uninformed observer with the impression that all international standards are roughly equal. Given CIOMS's historic position with respect to conflicts of interest in human subjects research, and given its prominent position in the international health community, it should lead that community in the direction of stronger standards for conflicts of interest. Yet, its past actions suggest that CIOMS may be unwilling to do so.

Likewise, the U.S. Food and Drug Administration (FDA) is uniquely positioned to force a discussion of stronger conflicts of interest standards at ICH. ${ }^{69}$ FDA is a member of $\mathrm{ICH}^{70}$ Additionally, the FDA has domestic experience with the need to strengthen conflicts of interest regulation and a public responsibility to protect human subject safety. Thus, the FDA is a prime candidate to open a new dialogue among ICH members concerning conflicts of interest. Yet, this would depend on the cooperation of other ICH members.

Alternatively, the FDA could refuse to approve a new drug for market unless all of the human subjects data supporting the marketability of the new drug were derived from human subject trials that complied with U.S. standards for managing conflicts of interest, which are stronger than those found in international guidelines. ${ }^{71}$ The strength of this strategy is that it uses access to the U.S. market as an inducement for drug companies to comply with stronger conflicts of interest standards and that it does not depend on the cooperation of the EU or Japan. This strategy will succeed, however, only if a significant change in policy is made by the FDA, which has singled out data from foreign drug trials for separate regulation pursuant to $\mathrm{ICH} \mathrm{GCPs}^{72}$ as a result of its commitment to the cooperative process of harmonizing domestic drug development laws among the U.S., EU and Japan.

Yet another strategy is for a non-governmental organization interested in protecting the interests of developing countries that host foreign clinical trials to propose guidelines that governments in those countries could employ as standards for approving foreign sponsored drug trials. The more experience governments in developing countries have with hosting human drug trials, the more likely they are to realize the risks associated with conflicts of interest in those trials, and the more ready they will become to protect their citizenry from those risks. Perhaps an international organization can form a coalition among such countries to uniformly strengthen their domestic laws against the risks associated with conflicts of interest from foreign-sponsored, human drug trials. Given the commercial interests of the

67 This may be as a result of its affiliation with WHO and WHO's interest in promoting international commerce in pharmaceuticals.

68 See generally CIOMS International Ethical Guidelines for Biomedical Research Involving Human Subjects, supra note 18.

69 See Gatter, supra note 4.

70 Justina Molzon, The International Conference on Harmonization Common Technical Document - Global Submission Format?, 60 FOOD \& DRUG L.J. 447, 448 (2005).

$71 \quad$ See 42 CFR $\S \S 50.603-605$ (2006); 21 CFR $\S \S 54.2,54.4-54.5$ (2006).

$72 \quad$ See 21 C.F.R. $\$ 312.120(2006)$. 
international pharmaceutical industry, the mere development of such a coalition might cause the industry to impose on itself sufficient conflicts of interest standards so as to avoid more protective action by governments of countries that routinely host foreign-sponsored studies. This strategy will likely fail, however, as long as there are a sufficient number of developing countries willing to ignore such guidelines so as to enjoy the short term financial benefits of hosting human subject trials. 